\title{
Interleukin-22 binding protein (IL-22BP) is constitutively expressed by a subset of conventional dendritic cells and is strongly induced by retinoic acid
}

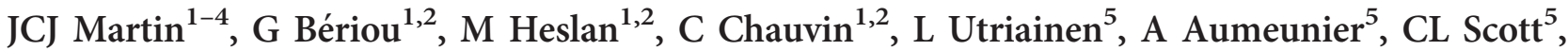

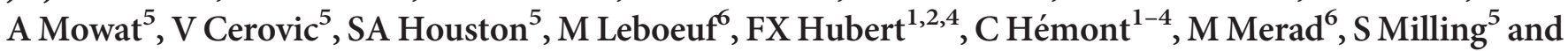 \\ R Josien ${ }^{1-4}$
}

Interleukin-22 (IL-22) is mainly produced at barrier surfaces by Tcells and innate lymphoid cells and is crucial to maintain epithelial integrity. However, dysregulated IL-22 action leads to deleterious inflammation and is involved in diseases such as psoriasis, intestinal inflammation, and cancer. IL-22 binding protein (IL-22BP) is a soluble inhibitory IL-22 receptor and may represent a crucial regulator of IL-22. We show both in rats and mice that, in the steady state, the main source of IL-22BP is constituted by a subset of conventional dendritic cells (DCs) in lymphoid and non-lymphoid tissues. In mouse intestine, IL-22BP was specifically expressed in lamina propria CD103 ${ }^{+}$CD11 b ${ }^{+}$DC. In humans, IL-22BP was expressed in immature monocyte-derived $\mathrm{DC}$ and strongly induced by retinoic acid but dramatically reduced upon maturation. Our data suggest that a subset of immature DCs may actively participate in the regulation of IL-22 activity in the gut by producing high levels of IL-22BP.

\section{INTRODUCTION}

Interleukin 22 (IL-22) is a member of the IL-10 cytokine family. ${ }^{1}$ IL-22 signals through the class 2 cytokine receptor family member (CRF2) IL-22 receptor (IL-22R) consisting of IL-10R $\beta 1$ and IL-22R1 chains. IL-22R1 expression is restricted to epithelial cells, hepatocytes, and acinar cells of the pancreas, ${ }^{2-4}$ while IL-10R $\beta 1$ expression is ubiquitous. By contrast, IL-22 is produced by a broad variety of immune cells. Innate sources of IL-22 are principally innate lymphoid cells (ILCs), ${ }^{1}$ mainly lymphoid tissue inducer (LTi)-like cells and the recently described ILC22, which shares characteristics with LTi and natural killer (NK) cells, and specialize in IL-22 production. Adaptive IL-22 sources are exclusively T lymphocytes. ${ }^{1}$

The IL-22/IL22R expression pattern makes IL-22 an important cytokine mediating the cross-talk between leucocytes and epithelia, particularly at barrier surfaces. Indeed, IL-22 is critical in reinforcing innate immune defences of epithelial cells in gut and lung infectious models. ${ }^{5,6} \mathrm{IL}-22$ is also important in the promotion of tissue repair. ${ }^{3,7-9}$ Nevertheless, numerous studies have also demonstrated that IL-22 can be a potent inducer of pathological inflammation. Indeed, IL-22 can promote tissue inflammation and self-destruction ${ }^{10-13}$ and is involved in the pathophysiology of several immune-mediated inflammatory diseases, such as psoriasis and rheumatoid arthritis. These paradoxical effects of IL-22 are dependent on the context of IL-22 production, ${ }^{13}$ as IL-22 can synergistically act with other inflammatory cytokines, including IL-17 and tumor necrosis factor- $\alpha \cdot{ }^{13,14} \mathrm{~A}$ tight regulation of the IL-22/IL-22R axis appears therefore critical in maintaining the beneficial effects of IL-22 and avoiding deleterious inflammatory effects.

\footnotetext{
${ }^{1}$ INSERM Center of Research in Transplantation and Immunology, UMR1064, Nantes, France. ${ }^{2} \mathrm{CHU}$ Nantes, Institut de Transplantation Urologie Néphrologie (ITUN), Nantes, France. ${ }^{3} \mathrm{CHU}$ Nantes, Laboratoire d'immunologie, Nantes, France. ${ }^{4}$ Faculté de Médecine, Université de Nantes, Nantes, France. ${ }^{5}$ Centre for Immunobiology, Institute of Infection, Immunity and Inflammation, College of Medical, Veterinary and Life Sciences, University of Glasgow, Glasgow, UK. ${ }^{6}$ Department of Gene and Cell medicine and the Department of Medicine, Mount Sinai School of Medicine, New York, New York, USA. Correspondence: R Josien (Regis.josien@univ-nantes.fr)
} 
IL-22 is the only member of the IL-10 cytokine family to have a soluble-secreted receptor, called IL-22 binding protein (IL-22BP, also known as IL22RA2). ${ }^{15-17}$ IL-22BP specifically binds to IL-2 $2{ }^{18}$ and is also a CRF2 member. Among the CRF2 members, IL-22BP shares the highest structural homology with the IL-22R1 chain. Nevertheless, IL-22BP exhibits a much higher affinity for IL-22 than the transmembrane IL-22R. ${ }^{19}$ Crystallization experiments revealed the molecular basis of the affinity differences between the IL-22/IL22BP and IL-22/ IL-22R1 complexes. ${ }^{20}$ IL-22BP inhibits IL-22 biological effects in vitro ${ }^{15-17}$ and in vivo ${ }^{8}$ and is constitutively expressed in secondary lymphoid organs (SLO), breast, and epithelial tissues, such as gut, lung, and skin. ${ }^{15}$ Taken together, these results suggest that IL-22BP acts as a natural regulator of IL-22, preventing exaggerated effects of the cytokine. Understanding IL-22BP biology may allow a better assessment of IL-22 paradoxical effects mentioned above. However, very little is known regarding IL-22BP cellular sources, regulation, and properties in vivo.

Dendritic cells (DCs) are rare cells of hematopoietic origin, widely distributed in the majority of tissues and specialized in capture, processing, and presentation of antigens to naive T cells. ${ }^{21}$ Depending on the type of DC and its activation state, the $\mathrm{DC} / \mathrm{T}$ cell interaction leads to the initiation of an immune response or to T-cell tolerance. DCs are classified as conventional, plasmacytoid, or inflammatory DCs. ${ }^{22}$ Conventional DCs (cDCs) include non-lymphoid resident tissue DCs that migrate to draining lymph nodes (LNs) and lymphoid resident DCs. Both are equipped with various pathogen-recognition receptors allowing them to sense the environment and recognize pathogen-associated molecular patterns. ${ }^{23}$ Before encountering a pathogen, DCs are present in an immature state and specialize in sampling the environment. After pathogenic antigen capture and recognition, cDCs undergo maturation and express high levels of major histocompatibility complex (MHC) and co-stimulatory molecules, allowing them to activate naive $\mathrm{T}$ cells. By this process, $\mathrm{cDCs}$ are able to elicit specific adaptive immune responses making them the necessary link between innate and adaptive immunity. In the absence of maturation signals, cDCs present antigens in an immature state, leading to T-cell tolerance. cDCs therefore also contribute to the maintenance of peripheral tolerance to self-antigens.

In this study, we show that the constitutive production of IL-22BP in lymphoid and gut tissues results from a production by a subset of $\mathrm{cDCs}$ at an immature state. We identify retinoic acid (RA) as a potent inducer of IL-22BP expression in DCs and show that IL-22BP expression is dramatically downregulated upon DC maturation.

Our results suggest a new role for $\mathrm{CDCs}$ in the maintenance of tissue homeostasis by participating in the IL-22-mediated dialogue between immune and epithelial cells.

\section{RESULTS}

\section{Tissue expression pattern of IL-22BP in the rat}

We first assessed the expression of IL-22BP mRNA in rat tissues and organs. The highest levels of IL-22BP were found in SLO,
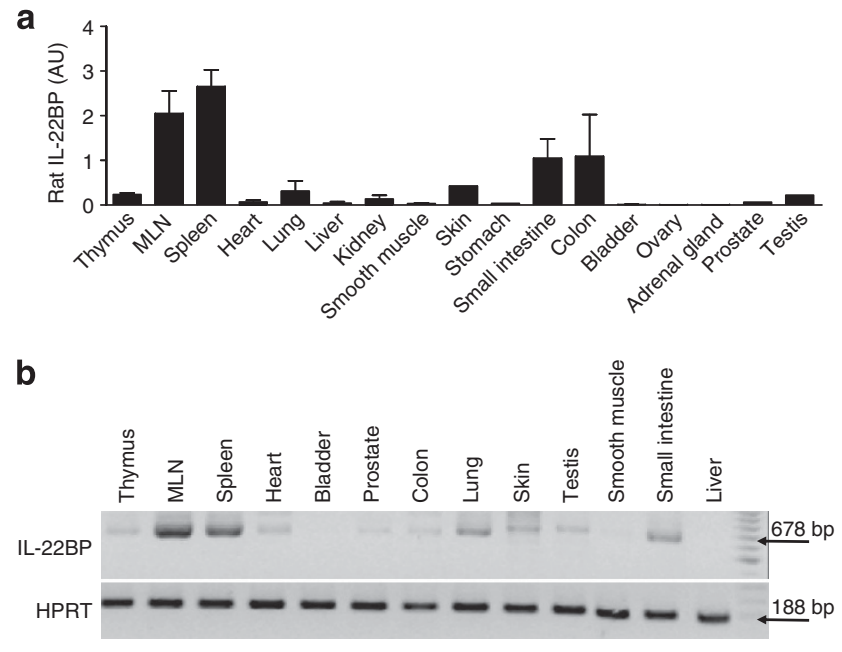

Figure 1 Tissue expression pattern of interleukin-22 binding protein (IL22BP) in the rat. (a) IL-22BP gene expression was analyzed by quantitative reverse transcriptase-PCR (RT-qPCR). Bars represent the mean \pm s.e.m. ratio of IL-22BP gene to HPRT (hypoxanthine-guanine phosphoribosyltransferase) expression as determined by the $2^{-\Delta \Delta C t}$ method of relative quantification from two experiments. (b) IL-22BP gene expression was analyzed by RT-PCR using primers allowing amplification of whole il22ra2 mRNA. Values indicate expected molecular mass of PCR products. Data are representative of two independent experiments. AU, arbitrary units; MLN, mesenteric lymph node.

i.e., spleen and mesenteric, axillary and cervical LNs (Figure 1a and Supplementary Figure S1A online). Lower levels of expression were found in thymus and several epithelial tissues, including gut, lungs, skin, and testis. This expression pattern was in agreement with those reported in human and mouse. ${ }^{15,17,24}$ However, although we confirmed that IL-22BP mRNA was strongly expressed in LN in mouse, it was expressed at very low levels in spleen (Supplementary Figure S1B), suggesting species- or strain-specific variation in the pattern of IL-22BP expression.

Three isoforms of IL-22BP exist in human ${ }^{15-17}$ but only isoform 2 efficiently binds and inhibits IL-22. ${ }^{15,16}$ The physiological roles of the other isoforms remain undetermined. Interestingly, only the human isoform 2 counterpart was identified in mouse, ${ }^{24}$ suggesting that IL-22 regulation by IL-22BP is an evolutionarily conserved process. Only one band, corresponding to human isoform 2, was detected in rat tissues (Figure 1b). The absence in the rat il22ra2 gene of a sequence corresponding to human exon $4 \mathrm{a}^{16}$ suggested that isoform $1 \mathrm{did}$ not exist in rat. In agreement with this observation, human isoform 1 was shown to be the result of a long terminal repeat insertion in the ape lineage. ${ }^{25}$ Finally, rat counterpart of human isoform 3 was not detected in these samples.

\section{IL-22BP is highly expressed in rat spleen by a subset of resident $\mathrm{CDCs}$ in the steady state}

High expression of il22ra2 gene in SLO suggested a hematopoietic origin of IL-22BP cellular sources. To test this hypothesis, different populations of hematopoietic cells were isolated from rat spleen by cell sorting, including the three 

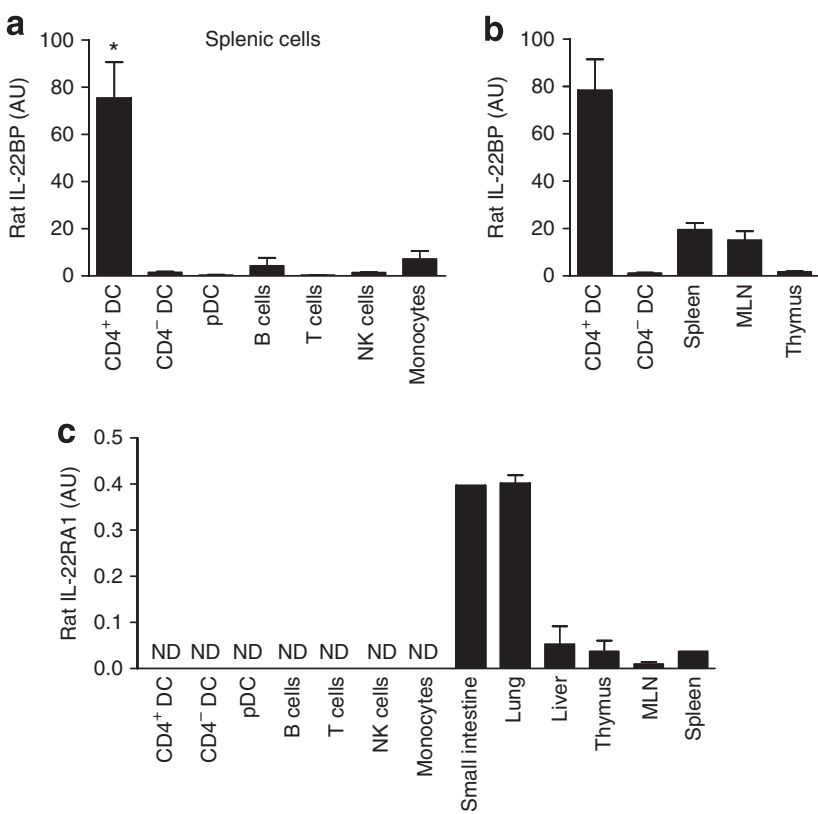

Figure 2 Interleukin-22 binding protein (IL-22BP) is highly expressed in the spleen by a subset of resident conventional dendritic cells (DCs). (a) The different populations of splenic hematopoietic cells were isolated by cell sorting. IL-22BP gene expression was analyzed by quantitative reverse transcriptase-PCR (RT-qPCR) and normalized relative to HPRT (hypoxanthine-guanine phosphoribosyltransferase) expression ( $n=4$ for DCs; $n=5$ for B cells; $n=3$ for T cells, natural killer (NK) cells, and monocytes). (b) IL-22BP gene expression was compared by RT-qPCR for DCs $(n=3)$ in spleen, mesenteric lymph node (MLN), and thymus $(n=2)$. In $\mathbf{a}$ and $\mathbf{b}$, bars represent mean \pm s.e.m. of IL-22BP gene to HPRT expression as determined by the $2^{-\Delta \Delta C t}$ method of relative quantification. (c) Hematopoietic cells were isolated by cell sorting. IL-22R1 gene expression was analyzed by RT-qPCR with several tissues being used as positive controls ( $n=3$ for cell samples; $n=2$ for tissue samples). Bars represent mean \pm s.e.m. ratio of IL-22R1 gene to HPRT expression as determined by the $2^{-\Delta \Delta C t}$ method of relative quantification. ${ }^{*} P<0.05$. AU, arbitrary units; ND, not detected; pDC, plasmacytoid dendritic cell.

subsets of DCs we have previously described (plasmacytoid DCs, CD172a ${ }^{+} \mathrm{CD}^{+}$, and $\left.\mathrm{CD} 172 \mathrm{a}^{-} \mathrm{CD} 4^{-} \mathrm{cDCs}\right) .^{26,27}$ Quantitative PCR (qPCR) analysis revealed high IL-22BP expression by the $\mathrm{CD} 172 \alpha^{+} \mathrm{CD} 4^{+} \mathrm{cDC}$ subset (referred thereafter as $\mathrm{CD}^{+}{ }^{+}$splenic DCs (spDCs)), whereas other populations only showed low levels of expression (Figure 2a). Moreover, $\mathrm{CD}^{+}{ }^{+}$spDCs presented a fivefold higher expression of IL-22BP than whole spleen (Figure 2b), strongly suggesting that these cells are the major source of IL-22BP in spleen in the steady state.

IL-22BP protein expression was evaluated by immunofluorescence staining of sorted DCs. IL-22BP staining was detected in virtually all $\mathrm{CD} 4{ }^{+}$spDCs, whereas fluorescence observed in $\mathrm{CD}^{-}{ }^{-}$spDCs was not different from that obtained after staining with secondary antibody only (Figure 3).

Finally, we did not detect any expression of the IL-22RA1 transmembrane receptor in the various splenic hematopoietic cell subsets, including $\mathrm{CD}^{+}{ }^{+}$spDCs (Figure 2c).

Taken together, our data suggest a role for $\mathrm{CD} 4{ }^{+}$spDCs in the regulation of IL-22 biological effects in the steady state via IL-22BP secretion.

\section{A subset of intestinal DCs expresses high levels of IL-22BP in the steady state}

We hypothesized that cDCs might also be a constitutive source of IL-22BP in epithelial tissues, and particularly in the gut where significant levels of IL-22BP expression were detected (Figure 1). We first analyzed IL-22BP expression by migrating intestinal rat $\mathrm{CDC}$ subsets. For this purpose, intestinal lymph DCs (ilDCs) were obtained by lymph collection after thoracic duct cannulation of mesenteric lymphadenectomized rats. ${ }^{28} \mathrm{MHCII}{ }^{\mathrm{hi}} \mathrm{CD} 103^{+}$lymph DC subsets were then sorted into three subsets based on their differential expression of CD172a and CD11b, as previously described ${ }^{28,29}$ High levels of IL-22BP mRNA expression were found in the CD172 $\mathrm{a}^{\text {high }}$ ilDC subset (referred thereafter as CD172a $\mathrm{a}^{\text {high }}$ ilDCs). This expression was about 50-fold higher than IL-22BP expression in small intestine (SI) and colon (Figure 4a). High expression of IL-22BP was also observed in CD172a ${ }^{+} \mathrm{CD} 4{ }^{+}$DCs from rat mesenteric LNs (MLNs; Figure $\mathbf{4 b}$ and Supplementary Figure S2) and Peyer patches (data not shown). In addition, in vitro DC depletion abolished IL-22BP expression in rat MLN (Figure 4c).

Interestingly, detailed phenotypical, functional, and transcriptomic analysis of CD172 $\mathrm{a}^{\text {high }}$ ilDCs vs. CD172a $\mathrm{a}^{+} \mathrm{CD} 4^{+}$ spDCs indicated that they have very strong similarities (R Josien and S Milling, unpublished observations) and most likely represent the same DC subset. This suggests that high IL-22BP expression is an intrinsic property of this subset of cDCs. Of note, IL-22BP expression was fivefold higher in CD172 $\mathrm{a}^{\text {high }}$ ilDCs than in CD4 ${ }^{+}$spDCs (Figure 4a). Thus, even if IL-22BP expression is a characteristic of these cells, factors present in the gut environment are also likely to promote higher levels of IL-22BP expression.

To determine whether such a functional specialization of CDC subsets regarding IL-22BP expression also holds true in mice, we first analyzed data from mouse cDNA microarrays obtained from the Immunological Genome Project (available from the ImmGen website: www.immgen.org). High levels of IL-22BP expression in steady state were observed in one small intestinal lamina propria (LP) DC subset, namely $\mathrm{CD} 103^{+}$ DCs. We confirmed IL-22BP expression by the LP CD103 ${ }^{+}$ $\mathrm{CD}_{11 \mathrm{~b}^{+}}$subset by qPCR (Figure 4d). By contrast, only low levels could be detected in $\mathrm{CD} 103^{+} \mathrm{CD} 8 \alpha^{+}$LPDCs and intestinal LP macrophages. In MLN, MHCII ${ }^{\text {high }} \mathrm{CD} 03^{+} \mathrm{DCs}$, which are known to be migrating DCs, ${ }^{30}$ were sorted in $\mathrm{CD} 11 \mathrm{~b}^{+}$ and $\mathrm{CD} 11 \mathrm{~b}^{-}$DCs; IL-22BP expression was much stronger in $\mathrm{CD}_{11 \mathrm{~b}^{+}}$DCs as compared with CD11b $\mathrm{b}^{-}$(Figure 4e). Moreover, IL-22BP expression was also significantly diminished in the colon of mice deficient for Fms-like tyrosine kinase 3 (Flt3L), which are known to lack $\mathrm{CD}_{103}{ }^{+}$DC (Figure 4f). Unexpectedly, SI from Flt3 $\mathrm{L}^{-1-}$ mice expressed similar levels of IL-22BP mRNA as compared with wild-type mice.

Finally, no IL-22BP expression was found among the different populations of human peripheral blood cells, including polymorphonuclear neutrophils, eosinophils, and basophils, lymphocytes, monocytes, as well as BDCA1 ${ }^{+}$and BDCA3 ${ }^{+}$ cDCs and plasmacytoid DCs (data not shown). 


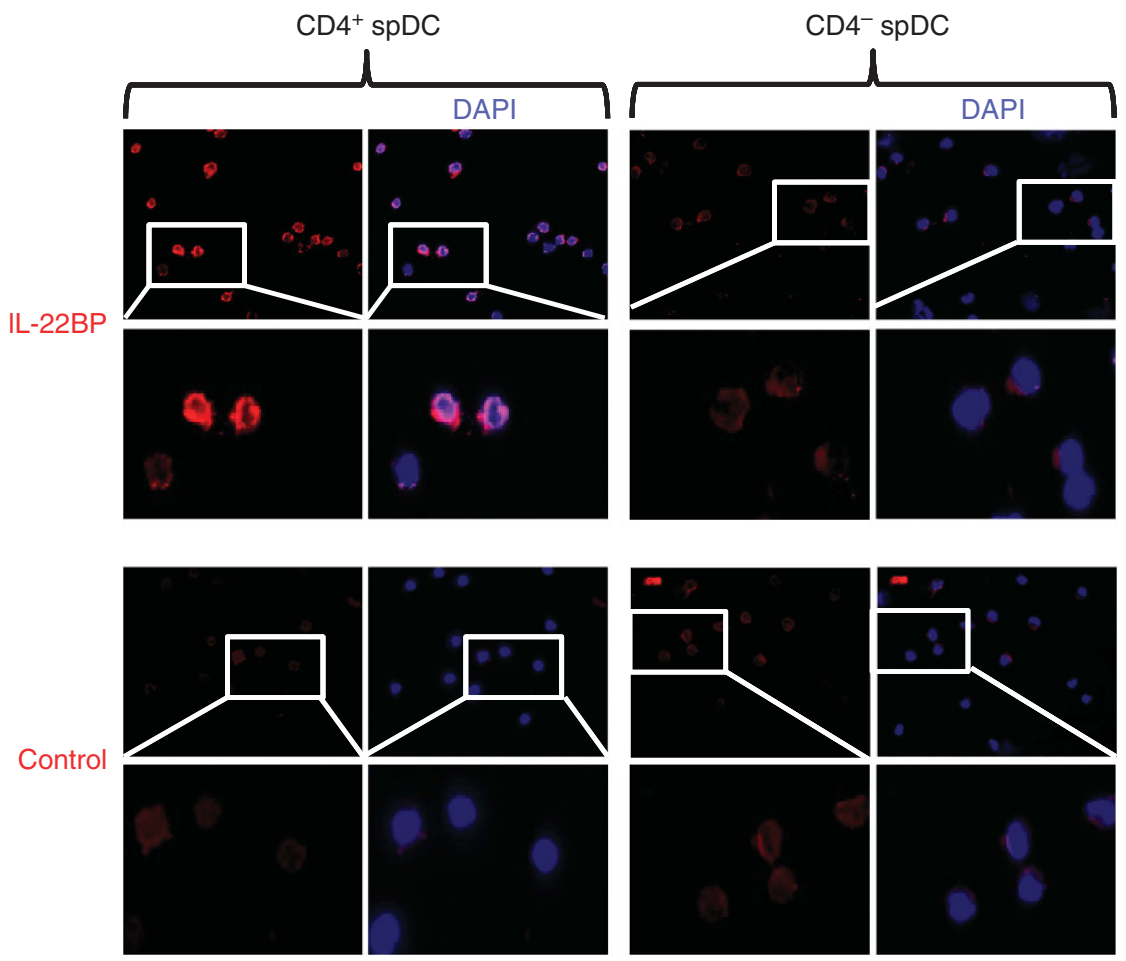

Figure 3 Interleukin-22 binding protein (IL-22BP) staining on sorted subsets of rat splenic conventional DCs. The two subsets of rat conventional spleen DCs (spDCs)were isolated by cell sorting and let to adhere on poly-L-Lysine pre-coated slides for 30 min in the presence of Brefeldin A and monensin. Slides were then stained with a goat anti-rat IL-22BP pAb followed by a donkey anti-goat immunoglobulin G-Alexa fluor 568 . Controls were performed using the secondary antibody alone. Data are representative of two independent experiments. DAPI, 4,6-diamidino-2-phenylindole.

Taken together, our results highly support the hypothesis that natural production of IL-22BP results mainly from a constitutive expression by a subset of cDCs both in SLO and in epithelial tissues.

\section{Rat CD4 ${ }^{+}$spDCs express a counterpart of the human short isoform of IL-22BP}

PCR experiments revealed the presence of two transcripts in rat $\mathrm{CD}^{+}{ }^{+}$spDCs (Figure 5), the longest and the most intense one corresponding to the expected $678 \mathrm{bp}$ mRNA, as further confirmed by sequencing. Translation of the nucleotide sequence obtained after sequencing gave a putative protein of 229 AA (data not shown), showing $72 \%$ identity with the human isoform 2 of IL-22BP, i.e., the isoform inhibiting IL-22 effects. Interestingly, sequencing of the PCR product corresponding to the shorter band identified a shorter isoform of rat IL-22BP. Like in human, the sequence of this isoform presented a complete excision of exon 5. Alignment of the rat short isoform with its human counterpart revealed $80 \%$ identity (Supplementary Figure S3A). Translation of the sequence gave a putative protein of $135 \mathrm{AA}$, showing $73 \%$ identity with human isoform 3 (Supplementary Figure S3B). Expression of this shorter isoform probably results from an alternative mRNA splicing and its physiological significance is unknown. Two important residues for IL-22 binding, Y65 and R117, which are the rat counterparts of human Y67 and R119, are conserved in this short isoform. These two residues have been demonstrated to be independently crucial for IL-22 binding, ${ }^{20}$ suggesting that the rat IL-22BP short isoform may also be able to bind IL-22, although probably less efficiently.

\section{IL-22BP is expressed in human monocyte-derived DCs}

To study in more detail the regulation of IL-22BP expression in DCs, we analyzed its expression in monocyte-derived dendritic cells (MDDCs). As shown in Figure 6a,b. IL-22BP mRNA expression was strongly induced during DC differentiation. IL-22BP expression at the protein level was confirmed by indirect immunofluorescence stainings experiments (Figure 6c). Moreover, no clear IL-22BP induction was detected when monocytes were differentiated in other conditions than granulocyte macrophages colony-stimulating factor (GM-CSF) and IL-4-supplemented media (Supplementary Figure S4). Finally, we found that both IL-22BP isoforms 1 and 2 were expressed by MDDCs (Figure 6d) as further confirmed by sequencing (data not shown). Isoform 3 was not detected in human MDDCs.

\section{RA is a potent inducer of IL-22BP expression by DCs}

As IL-22BP expression was fivefold higher in rat intestinal DCs than in splenic DCs (Figure 4a), we hypothesized that inducers of IL-22BP expression could be present in the gut environment. We thus analyzed IL-22BP expression by MDDCs cultured with various molecules present in high amounts in the gut. 


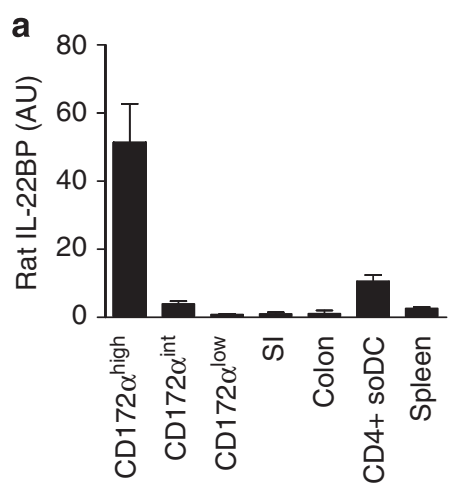

iIDC

\section{d}

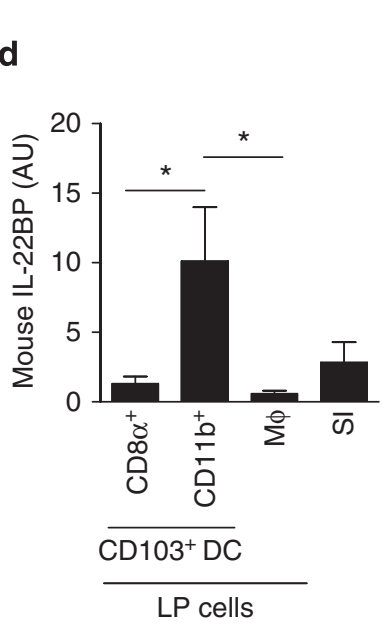

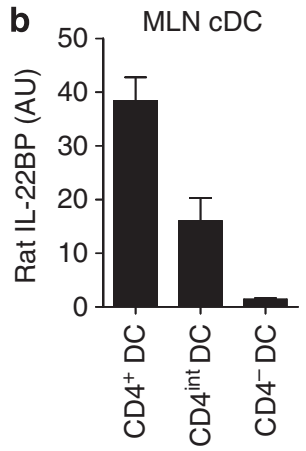

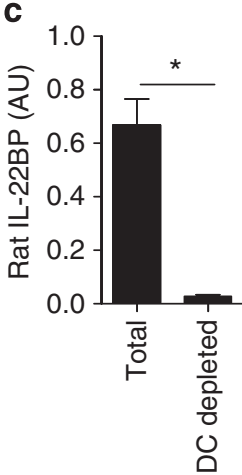

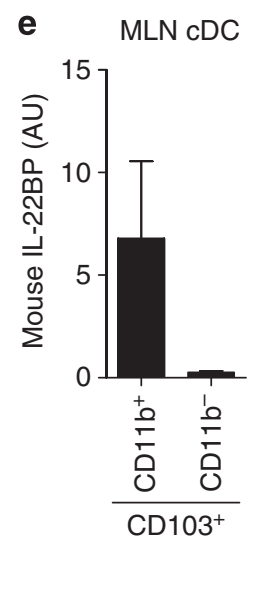

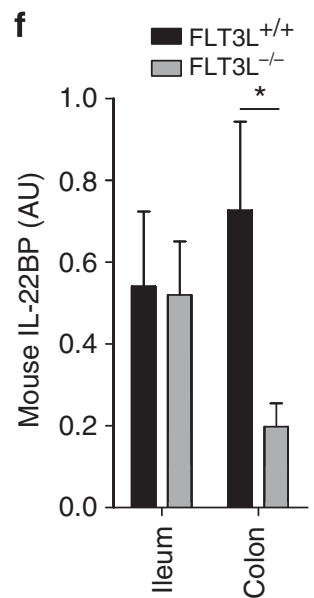

Figure 4 A subset of intestinal dendritic cells (DCs) expresses high levels of interleukin-22 binding protein (IL-22BP) in the steady state.

(a) Constitutively migrating intestinal lymph DCs (ilDCs) subsets were obtained by lymph collection after thoracic duct cannulation of mesenteric lymphadenectomized rat and cell sorting. IL-22BP gene expression was analyzed by quantitative reverse transcriptase-PCR (RT-qPCR) for the three subsets of ilDCs and compared with intestinal tissues and its CD4 ${ }^{+}$DC spleen counterpart ( $n=3$ for ilDCs; $n=2$ for tissues). (b) The three subpopulations of rat mesenteric lymph node (MLN)-DCs were isolated by cell sorting and analyzed for IL-22BP expression by RT-qPCR ( $n=3$ ). (c) Total DCs were depleted from total MLN cells by fluorescence-activated cell sorter depletion of CD103 ${ }^{+}$cells. IL-22BP gene expression was analyzed by RT-qPCR $(n=3)$. (d) Mouse CD8 ${ }^{+}$and CD11b ${ }^{+} \mathrm{CD}_{103^{+}} \mathrm{DC}$ subsets and macrophages were isolated from intestinal lamina propria (LP) by cell sorting. IL-22BP gene expression was analyzed by RT-qPCR ( $n=3$ for DCs and small intestine (SI) tissue; $n=2$ for macrophages (MФ)). (e) Mouse MLN CD103 ${ }^{+}$DCs were separated into CD11 ${ }^{+}$and CD11 b ${ }^{-}$by cell sorting and analyzed for IL-22BP expression by RT-qPCR $(n=3)$. (f) IL-22BP gene expression was analyzed by RT-qPCR in SI and colon of wild-type $(n=3)$ and Flt3L ${ }^{-1-}$ (Fms-like tyrosine kinase 3; $n=3$ ) mice. In $\mathbf{a}-\mathbf{c}$ (rat), e and $\mathbf{f}$ (mouse), bars represent mean \pm s.e.m. ratio of IL-22BP gene to HPRT (hypoxanthine-guanine phosphoribosyltransferase) expression as determined by the $2^{-\Delta \Delta C t}$ method of relative quantification. In $\mathbf{d}$ (mouse), bars represent mean \pm s.e.m. ratio of IL-22BP gene to GAPDH (glyceraldehyde 3-phosphate dehydrogenase) expression as determined by the $2^{-\Delta \Delta C t}$ method of relative quantification. ${ }^{*} P<0.05$. AU, arbitrary units; cDC, conventional dendritic cell.

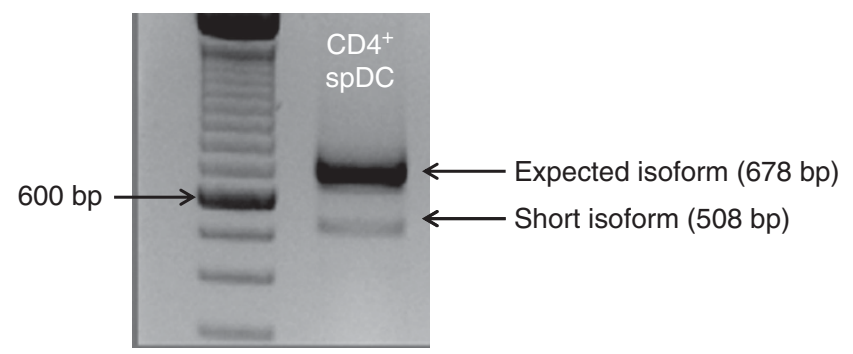

Figure 5 Rat $\mathrm{CD} 4{ }^{+}$dendritic cells (DCs) express a counterpart of the human short isoform of interleukin-22 binding protein (IL-22BP). Spleen $\mathrm{CD}^{+}{ }^{+}$conventional DCs were isolated by cell sorting. IL-22BP gene expression was analyzed by reverse transcriptase-PCR using primers allowing amplification of whole il22ra2 mRNA. spDC, spleen dendritic cell.
When added during the last 2 days of DC culture, transforming growth factor- $\beta$ (TGF- $\beta$ ), thymic stromal lymphopoietin, IL-13, and IL-10 had no effect on IL-22BP mRNA expression (Figure 7). By contrast, a strong upregulation (eightfold) of IL-22BP expression was induced by AM580, an agonist of RA receptor alpha (RAR $\alpha$ ) (Figure 7), suggesting that RA can enhance IL-22BP expression in DCs.

The effects of the RAR $\alpha$ agonist AM580 were even stronger when added on day 0 of MDDC differentiation. AM-DCs (AM580 added on d0) exhibited a strong upregulation of IL-22BP mRNA and protein expression (Figure 8a-c). Interestingly, AM-DCs not only upregulated IL-22BP expression but also acquired a different phenotype characterized 


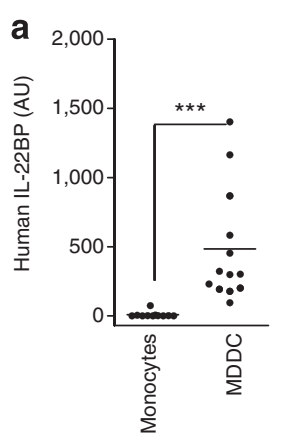

\section{b}

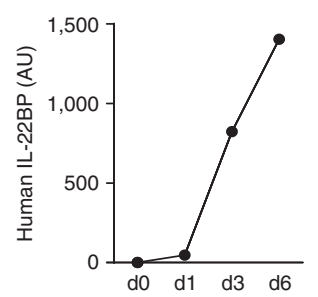

C

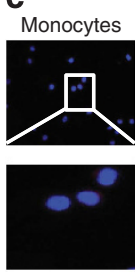

IL-22BP

DAPI

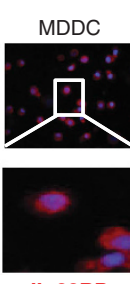

IL-22BP DAPI

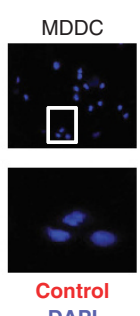

DAPI d

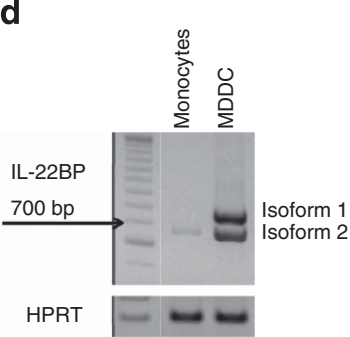

Figure 6 Interleukin-22 binding protein (IL-22BP) is expressed in human monocyte-derived dendritic cells (MDDCs). Human monocytes from peripheral blood of healthy donors were differentiated into DCs in complete medium with granulocyte macrophages colony-stimulating factor and IL-4 for 6 days. (a) On day 6, IL-22BP gene expression was analyzed by quantitative reverse transcriptase-PCR (RT-qPCR). Each point represents the ratio of IL-22BP gene to HPRT (hypoxanthineguanine phosphoribosyltransferase) expression, as determined by the $2^{-\Delta \Delta C t}$ method of relative quantification, for an individual healthy donor $(n=14)$. (b) Cells were collected at the indicated times during MDDC differentiation and IL-22BP gene expression analyzed by RT-qPCR. Each point represents the ratio of IL-22BP gene to HPRT expression as determined by the $2^{-\Delta \Delta C t}$ method of relative quantification. Data are representative of two independent experiments. (c) Monocytes and MDDCs were let to adhere on poly-L-Lysine pre-coated slides for $30 \mathrm{~min}$ in the presence of Brefeldin $A$ and monensin and then stained with a mouse immunoglobulin G1 (IgG1) anti-human IL-22BP as primary antibody followed by goat anti-mouse IgG1-Alexa fluor 568. Controls were performed using the secondary antibody alone. Data are representative of four independent experiments. (d) IL-22BP gene expression was analyzed by RT-PCR using primers allowing amplification of whole il22ra2 mRNA. Data are representative of three independent experiments. ${ }^{\star * \star} P<0.001$. DAPI, 4,6-diamidino-2-phenylindole.

by CD103 and CD1d expression, and loss of CD1a expression (Figure 8d), confirming previously published results. ${ }^{31,32}$ The same results were obtained when RA itself was added in the culture (Figure 8e and data not shown). Moreover, experiments using selective inhibitors of RA nuclear receptor revealed that RA effects on IL-22BP, CD103, and CD1d expression induction by MDDCs mostly involved RAR $\alpha$ signaling (Figure 8e and data not shown).

RA is the active metabolite of dietary vitamin A. Vitamin A is first oxidized into retinal by several enzymes, including alcohol dehydrogenase, then retinal is oxidized into RA by specific dehydrogenases, including retinal dehydrogenase 2 (RALDH2). ${ }^{33}$ GM-CSF and IL-4 induce the expression of RALDH2 in human MDDCs ${ }^{34}$ early during differentiation (Supplementary Figure S5A,B), which endows them with the

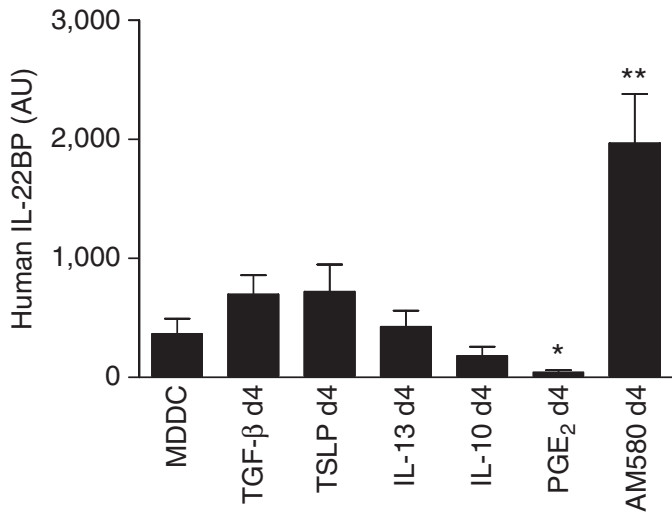

Figure 7 Retinoic acid is a potent inducer of interleukin-22 binding protein (IL-22BP) expression by dendritic cells (DCs). Human monocytes from peripheral blood of healthy donors were differentiated into DCs in complete medium with granulocyte macrophages colony-stimulating factor and IL-4 for 6 days. When indicated, ligands were added at day 4 of culture. On day 6, IL-22BP gene expression was analyzed by quantitative reverse transcriptase-PCR. Bars represent mean \pm s.e.m. ratio of IL-22BP gene to HPRT (hypoxanthine-guanine phosphoribosyltransferase) expression as determined by the $2^{-\Delta \Delta \mathrm{Ct}}$ method of relative quantification from seven independent experiments ${ }^{\star \star} P<0.01 ;{ }^{\star} P<0.05$. MDDC, monocyte-derived dendritic cell; $P \mathrm{PE}_{2}$, prostaglandin $\mathrm{E}_{2}$; TGF, transforming growth factor; TSLP, thymic stromal lymphopoietin.

capacity to metabolize retinal into RA. IL-22BP expression was upregulated by addition of retinal during DC differentiation. This induction was significantly diminished by a RALDH2 reversible inhibitor, diethylaminobenzaldehyde (DEAB; Figure 8f). These findings implied that specific metabolism of retinal by human MDDCs allows RA production, which in turn induces IL-22BP expression, probably in an autocrine fashion.

Interestingly, addition of $\mathrm{PGE}_{2}$ (prostaglandin $\mathrm{E}_{2}$ ), which was recently described as a RALDH2 inhibitor, ${ }^{34}$ abolished the expression of IL-22BP in MDDCs (Figure 7). This suggests that constitutive expression of IL-22BP by MDDCs could be due to low levels of RA production, from retinal present in the serum added in the culture media. However, MDDCs differentiated in serum-free media or in serum-supplemented media with the RAR $\alpha$ antagonist also expressed IL-22BP (data not shown and Supplementary Figure S5C), indicating that IL-22BP production is not totally dependent on DC's ability to produce RA.

RALDH2 expression in MDDCs peaked after $20 \mathrm{~h}$ and progressively decreased until day 6 (Supplementary Figure S5B), which could suggest that RA exerts its effects on MDDCs at early stages of the differentiation. We therefore examined the effect of delayed addition of the RAR $\alpha$ inhibitor on MDDC differentiation in the presence of RA. CD103 induction was inhibited when the RAR $\alpha$ inhibitor was added at days $0,1,2$, and 3 of the culture but not later (Supplementary Figure S5D). The effects on CD1d were even more pronounced as inhibition was only observed when the inhibitor was added at the beginning of the culture. This indicated that RA effects on the AM-DC phenotype occur early during the differentiation. 
a

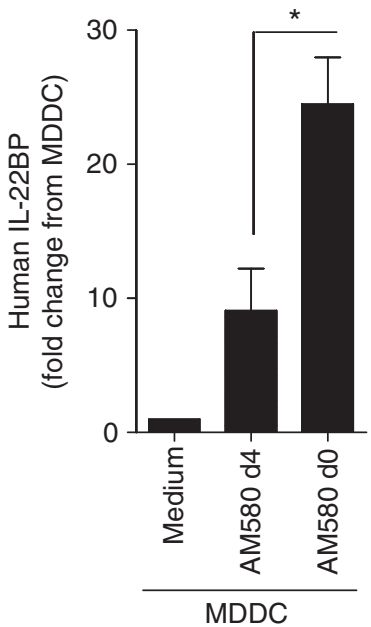

b

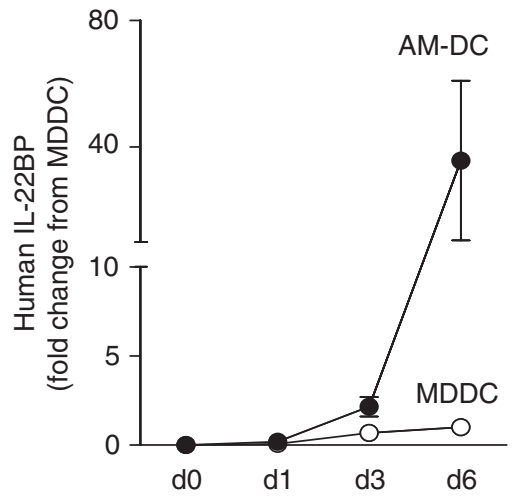

C
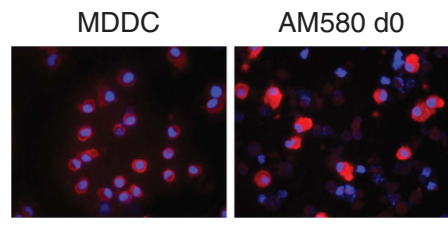

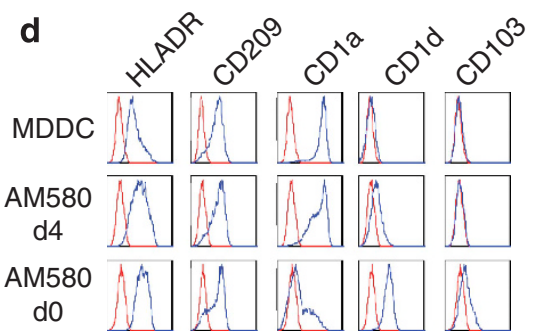

g e

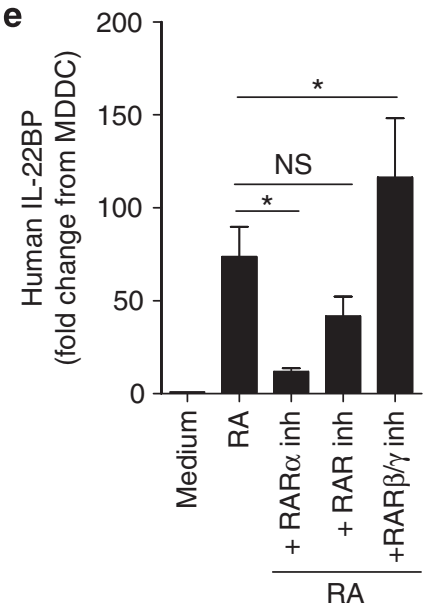

f

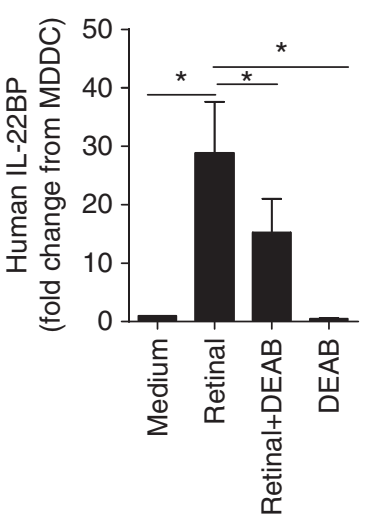

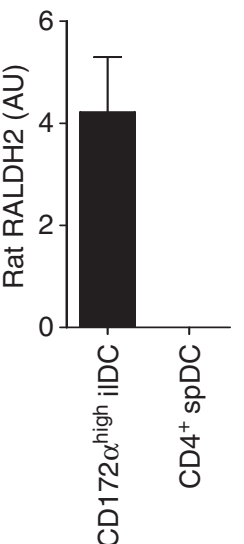

Figure 8 AM580-induced differentiation of monocyte-derived dendritic cells (MDDCs) enhances interleukin-22 binding protein (IL-22BP) expression. (a) MDDCs were differentiated in the absence or presence of AM580 added at day 0 or day 4 of culture. IL-22BP gene expression was analyzed by quantitative reverse transcriptase-PCR (RT-qPCR). Bars represent mean \pm s.e.m. of fold change compared with GM-CSF (granulocyte macrophages colony-stimulating factor)/IL-4-derived MDDCs, of IL-22BP gene to HPRT (hypoxanthine-guanine phosphoribosyltransferase) expression as determined by the $2^{-\Delta \Delta \mathrm{Ct}}$ method of relative quantification $(n=4)$. (b) Cells were harvested at the indicated times during MDDC differentiation in the presence or the absence of AM580 added at day 0. IL-22BP gene expression was analyzed by RT-qPCR. Each point represents mean \pm s.e.m. of fold change compared with GM-CSF/L-4-derived MDDCs, of IL-22BP gene to HPRT expression as determined by the $2^{-\Delta \Delta C t}$ method of relative quantification $(n=4)$. (c) MDDCs and AM580-diferentiated MDDCs were stained for IL-22BP as described in Figure 6. (d) MDDCs were differentiated in the absence or presence of AM580 added at day 0 (AM-DCs) or day 4 of culture. Cell surface markers were analyzed by flow cytometry. Red histograms, isotype control staining; blue histrograms, antibody staining. Data are representative of at least four independent experiments. (e) MDDCs were differentiated in the absence or presence of retinoic acid (RA) and specific inhibitors of RA nuclear receptors added at d0. IL-22BP gene expression was analyzed by RT-qPCR. Bars represent mean \pm s.e.m. of fold change, compared with GM-CSF/IL-4-derived MDDCs, of IL-22BP gene to HPRT expression as determined by the $2^{-\Delta \Delta \mathrm{Ct}}$ method of relative quantification ( $n=4$ ). (f) MDDCs were differentiated in the presence or not of retinal and/or diethylaminobenzaldehyde (DEAB), a selective inhibitor of RALDH2. IL-22BP gene expression was analyzed by RT-qPCR. Bars represent mean \pm s.e.m. of fold change, compared with GM-CSF/IL-4-derived MDDCs, of IL-22BP gene to HPRT expression as determined by the $2^{-\Delta \Delta \mathrm{Ct}}$ method of relative quantification $(n=3)$. (g) RALDH2 gene expression was analyzed by RT-qPCR in rat CD172 ${ }^{+}$CD4 ${ }^{+}$splenic DCs (spDCs) and CD172 high intestinal lymph DCs (ilDCs). Bars represent mean \pm s.e.m. of IL-22BP gene to HPRT expression as determined by the $2^{-\Delta \Delta \mathrm{Ct}}$ method of relative quantification $(n=3)$. ${ }^{*} P<0.05$. DAPI, 4,6-diamidino-2phenylindole; raldh2, retinal dehydrogenase 2; RAR $\alpha$, retinoic acid receptor alpha; RXR, retinoic X receptor. 
This likely reflects the effects of RA on blood pre-DCs entering the gut mucosa, which enter a specific differentiation program and consequently display unique functional properties, as confirmed by previous studies. By contrast, IL-22BP expression was inhibited even when the RAR $\alpha$ inhibitor was added at day 5 of the culture (Supplementary Figure S5E), indicating that RA-induced IL-22BP expression required continuous signaling through RAR.

Altogether, these data identify RA as a potent inducer of IL-22BP expression in DCs. This suggests that the higher expression of IL-22BP by rat CD172a ${ }^{+}$ilDCs as compared with $\mathrm{CD} 4{ }^{+}$spDCs is due to the ability of the former cells to produce RA. In agreement with this, aldh1a2 gene expression (encoding for RALDH2) was detected in ilDCs and not in spleen cDCs (Figure 8g). Thus, the higher levels of IL-22BP expression of rat intestinal DCs compared with their splenic counterpart are likely to be the consequence of a permanent exposure to RA, explained notably by their ability to produce it.

IL-22BP is downregulated when DCs undergo maturation IL-22BP expression appears to be a constitutive property of a subset of CDCs at an immature state in SLO and epithelial tissues. We then assessed whether IL-22BP expression was modulated upon DC maturation. Spontaneous maturation, which occurs when DCs are cultured without any stimulators, induced a rapid and dramatic downregulation of IL-22BP expression in rat $\mathrm{CD}^{+}{ }^{+}$spDCs and MLN-DCs (Figure 9a and Supplementary Figure S6). This downregulation persisted for up to $48 \mathrm{~h}$. Stimulation by $\mathrm{CpG}$ ODN2006, a ligand for Toll-like receptor 9 (TLR9), did not affect this downregulation of IL-22BP expression (Figure 9a). Similar results were obtained after stimulation of DCs by other TLR ligands (including ligands for TLRs 1-6, 7, and 8) (data not shown).

In human MDDCs and AM-DCs, lipopolysaccharide (LPS) and LPS + IFN $\gamma$ (interferon- $\gamma$ )-induced maturation was associated with a dramatic decrease of IL-22BP expression (Figure 9b). Similar results were obtained with ligands of TLR3 (polyinosinic-polycytidylic acid) or TLR5 (flagellin) (data not shown). Altogether, these data indicate that constitutive expression of IL-22BP by DCs at an immature state is lost upon DC maturation. This is concordant with previous studies describing a downregulation of IL-22BP expression in inflammatory conditions, notably in mouse DSS (dextran sodium sulfate)-induced colitis. ${ }^{8,19}$ Interestingly, AM580 could partly restore IL-22BP expression by rat $\mathrm{CD}^{+}{ }^{+}$spDCs after spontaneous maturation (Figure 9c), again supporting a role for RA as a positive regulator of IL-22BP expression. The incomplete restoration of IL-22BP expression in mature DCs probably reflects that RAR $\alpha$ signaling is not sufficient to fully counteract the maturation-induced downregulation of IL-22BP. Similar observations of RA and maturation impacts on IL-22BP expression could be made in mouse bone marrowderived DCs (Supplementary Figure S7), even if IL-22BP expression levels were low in this model.
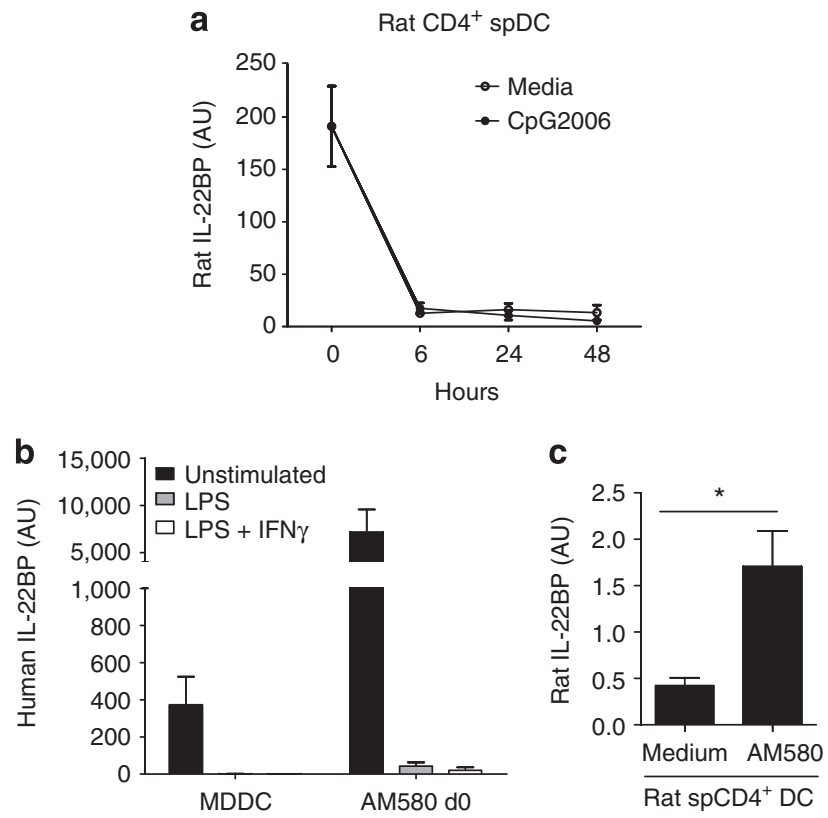

Figure 9 Interleukin-22 binding protein (IL-22BP) is downregulated when dendritic cells (DCs) undergo maturation. (a) Rat spleen CD4 ${ }^{+}$DCs were isolated by cell sorting and cultured for the indicated times in complete medium, in the absence or presence of the Toll-like receptor 9 (TLR9) ligand CpG (cytidine-phosphate-guanosine) ODN2006. IL-22BP gene expression was analyzed by quantitative reverse transcriptase-PCR (RT-qPCR; $n=3$ ). (b) Lipopolysaccharide (LPS) or LPS + IFN $\gamma$ (interferon- $\gamma$ ) was added at day 6 on human MDDCs. IL-22BP gene expression was analyzed by RT-qPCR after $24 \mathrm{~h}(n=2)$. (c) Rat spleen $\mathrm{CD} 4{ }^{+}$conventional DCs were isolated by cell sorting and cultured for $24 \mathrm{~h}$ in the absence or presence of AM580. IL-22BP gene expression was analyzed by RT-qPCR $(n=3)$. Data are presented as mean \pm s.e.m. of ratios of IL-22BP gene to HPRT (hypoxanthine-guanine phosphoribosyltransferase) expression as determined by the $2^{-\Delta \Delta C t}$ method of relative quantification (Rat, a and c; Human, b). AU, arbitrary units; MDDC, monocyte-derived dendritic cell; spDC, splenic DC.

\section{DISCUSSION}

Since its first description in 2000, the actions of IL-22 in mediating the cross-talk between the immune and epithelial systems were extensively described. By its ability to reinforce innate immunity of epithelial cells, notably by inducing antimicrobial peptide production, IL-22 has been demonstrated to be crucial in the resolution of Enterobacteriaceae gut and lung infections. ${ }^{5,6}$ Combined with the capacity of IL-22 to promote tissue repair in several inflammatory conditions, $3,7-9$ IL-22 properties seem necessary in the maintenance of tissue homeostasis. However, excessive actions of IL-22 may be particularly deleterious for epithelial tissues, ${ }^{10-12}$ suggesting that IL-22 needs to be tightly regulated to prevent unwarranted tissue damage and dysregulated epithelial cell proliferation. This regulation may be partly achieved by IL-22BP, given its specific inhibitory properties on IL-22 actions. ${ }^{8,15-17}$

Our study strongly suggests that the natural production of IL-22BP, observed in SLO and epithelial tissues, may be principally achieved by a subset of cDCs. These data extend the recent report by Huber et al. ${ }^{35}$ which showed in mice that IL-22BP-producing cells in colon were mainly $\mathrm{MHCII}^{+}$ 
CD11c ${ }^{+}$cDCs. IL-22BP production by rat spCD ${ }^{+} \mathrm{cDCs}$ was observed at an immature state, whereas maturation induces a dramatic downregulation of IL-22BP expression. Consistent with previous studies, ${ }^{8,19}$ these results suggest that IL-22BP production is prominent in the steady state, but not in inflammatory conditions, which lead to DC maturation. Nevertheless, IL-22BP upregulation has been observed in several infectious models, particularly in acute polymicrobial peritonitis, and after orally administration of Toxoplasma gondii. ${ }^{36,37}$ Interestingly, IL-22 was shown to be detrimental during these processes and led to uncontrolled deleterious inflammation. This suggests that control of IL-22 effects requires a tight regulation of IL-22BP production by cDCs. cDCs may produce high levels of IL-22BP at physiological state, when IL-22 actions are not desired, then, depending on the stimuli, IL-22BP production could be further enhanced or stopped. In our study, we could not induce an upregulation of IL-22BP expression by stimulating spCD $4^{+} \mathrm{cDCs}$ with several TLR ligands. In addition, inflammasome activation was recently shown to have an important role in the downregulation of IL-22BP expression in mouse colon during inflammation. ${ }^{35}$ Moreover, in vitro spontaneous maturation of $\mathrm{cDCs}$ and MDDCs also downregulated IL-22BP expression. Spontaneous maturation of mouse BMDCs was shown to result from the disruption of E-cadherin-mediated adhesion of DCs within each other, inducing the activation of the $\beta$-catenin signaling pathway. ${ }^{38}$ This process is characterized by a specific transcriptional profile and supposed to favour the generation of tolerogenic DCs in vivo. However, during the early phase of maturation, spontaneous and TLRs matured DCs exhibit a similar expression profile. Because IL-22BP is rapidly and equally downregulated in spontaneously and TLR-matured DCs (Figure 9a), it is likely that the il22ra2 gene belongs to this common program of matured DCs. Consequently, it remains to determine which cells can produce IL-22BP under inflammatory conditions.

The significance of the high levels of IL-22BP in SLO remains elusive. Indeed, only low levels of IL-22R1 expression are found in SLO. Moreover, IL-22 is not likely to act on immune cells in SLO as they do not express IL-22R1 at all $^{2}$ (Figure 2c). One possible explanation could be that the constitutive expression of IL-22BP by resident cDCs, which are highly represented in SLO, reflects ontogeny-related specializations similar to epithelial tissue cDCs. Indeed, high IL-22BP expression in rat $\mathrm{CD} 4{ }^{+}$spDCs and $\mathrm{CD} 172 \mathrm{a}^{+}$iLDCs might reflect a specific gene signature of this particular subset, similar to other genes such as SIRP $\alpha$ or DCIR1 for instance. DC subsets are classified on the basis of distinct cell-surface markers and display a specific gene signature explaining some functional specializations. ${ }^{39}$ Although IL-22R1 is not expressed by normal immune cells, aberrant expression by transformed lymphocytes has been described. ${ }^{40}$ Actions of IL-22 on these cells contributed to the pathogenesis of mantle cell leukemia and anaplastic large cell lymphoma. ${ }^{40,41}$ Therefore, high IL-22BP production in SLO could be necessary to prevent IL-22 actions on tumor cells, thus limiting the installation of these malignancies.
Strikingly, IL-22BP expression was about fivefold higher in the rat intestinal CD172a ${ }^{\text {high }}$ iLDCs compared with their splenic counterparts, suggesting that the gut environment is likely to favour IL-22BP production by these cells. We found that RA was a potent inducer of IL-22BP expression in vitro in human MDDCs. Moreover, MDDCs can oxidize retinal into RA, due to their expression of RALDH2. ${ }^{34}$ De novo production of RA could further enhance IL-22BP expression by these cells, probably in an autocrine fashion.

Interestingly, in mouse, a subset of intestinal DCs expressing CD103 constitutively expresses RALDH2. This subset mainly resides in the SI-LP and constitutively migrates toward draining LNs. $^{30}$ Mouse intestinal CD103 ${ }^{+}$DCs derive from a DC precursor continuously seeding the SI-LP from the circulation. ${ }^{42}$ Differentiation of this precursor toward the $\mathrm{CD} 103^{+}$ DC subset is likely due to environmental factors present in the SI-LP, ${ }^{43}$ including RA itself.

Our data suggest that, in mouse, the $\mathrm{CD} 103^{+} \mathrm{CD}_{11 b^{+}}$ subset of $\mathrm{cDCs}$ represent a constitutive source of IL-22BP in the gut. Notably, although Huber et al. ${ }^{35}$ reported much lower expression of IL-22BP in SI vs. colon, we found similar expression in both segments. Our finding that IL-22BP expression was strongly reduced in large intestine from Flt3 $\mathrm{L}^{-1-}$ mice suggests that Flt3L-dependent $\mathrm{cDC}$ are indeed a major source of IL-22BP in large intestine that is consistent with data from Huber et al. ${ }^{35}$ The apparent normal expression of IL-22BP mRNA we found in SI from Flt3L-deficient mice (Figure 4f) was more surprising and suggests that IL-22BP could be expressed by Flt3L-independent DCs or by other cells than DCs in SI, either constitutively or due to compensation process. These hypotheses need to be tested in vivo. Rat CD172a high $i$ ilDCs, which express very high levels of IL-22BP, display similar properties to the mouse $\mathrm{CD} 103^{+}$DC subset, including CD103 expression, constitutive migration from the intestinal mucosa towards the draining mesenteric LNs, and constitutive expression of RALDH2 (Figure 8g). Therefore, the CD172 $\alpha^{\text {high }}$ ilDC subset is likely to be the rat counterpart of the mouse $\mathrm{CD}_{103}{ }^{+} \mathrm{CD}_{11 \mathrm{~b}}{ }^{+}$DCs. Further study will be required to identify IL-22BP protein-producing cells in the LP as well as in SLO, as we did not succeeded in staining tissue with commercially available $\mathrm{mAbs}$. In fact, there is no information in the current literature, including in the recent paper from Huber et al., ${ }^{35}$ about IL-22BP protein expression in vivo.

Maintenance of gut homeostasis is complex and implicates the establishment of equilibrium between the host and the commensal microbiota, while maintaining the ability to fight against invading pathogens. The gut immune system has an important role in this equilibrium and needs to be tightly regulated to avoid unbalanced reactions leading to inflammatory bowel diseases. Interestingly, the $\mathrm{CD}_{103}{ }^{+} \mathrm{DC}$ subset seems to actively participate in maintenance of the gut homeostasis due to its unique functional properties, conserved between mice and humans. ${ }^{42}$ These properties include the ability of imprinting gut-homing receptors on naive 
lymphocytes, the induction of $\mathrm{CD}^{+}{ }^{+}$naive $\mathrm{T}$ cells differentiation into FOX-P3 ${ }^{+}$(forkhead box P3) regulatory T cells, and the differentiation of immunoglobulin A-secreting B cells. All these properties result from the ability of these cells to efficiently produce RA from retinal. ${ }^{44}$

Regulation of inflammatory cytokine production is also crucial in the maintenance of gut homeostasis. High IL-22 production by LTi cells was observed in the mouse fetus and before weaning. ${ }^{45}$ This high production of IL-22, concomitant with IL-17, is thought to be necessary to promote a local proinflammatory milieu at birth enabling the containment of the colonizing microbiota and the selection of appropriate flora. ${ }^{46}$ After birth, downmodulation of IL-22 production is observed for both LTi and ILC22 cells, following the integration of negative signals from the commensal flora. ${ }^{45}$ Interestingly, intestinal DCs appear to be involved in this regulation by responding to microbiota-induced IL-25 produced by epithelial cells. ${ }^{45}$ IL-25-responding DCs could further moderate IL-22 production by ILCs, in a contact-dependent way. However, whether these DCs corresponded to the $\mathrm{CD} 103^{+}$subset was not analyzed in this study. Our results further suggests that $\mathrm{CD}_{103}{ }^{+}$DCs could also regulate IL-22 actions by constitutively producing high levels of its natural soluble inhibitory receptor, reinforcing the importance of DCs in intestinal immune regulation. ${ }^{44}$

Only two studies addressed the role of IL-22BP in vivo. In a mouse model of acute DSS colitis, Sugimoto et al. ${ }^{8}$ demonstrated that beneficial effects of IL-22 on epithelium regeneration were abolished in the presence of IL-22BP. This study was the first to demonstrate effects of IL-22BP on IL-22 actions described in vivo. However, IL-22BP was shown to be downregulated during the acute phase of colitis, when IL-22 is highly produced. Thus, the model remained artificial as IL-22BP overexpression was induced by a local gene-delivery system. Moreover, neither the cellular source of native IL-22BP nor its protein expression in vivo were addressed in this study. A second study was published during the reviewing process of our manuscript by Flavell and colleagues. In this paper, the authors used a model of IL-22BP ${ }^{-1-}$ mice. ${ }^{35}$ They confirmed the coordinated regulation of IL-22 and IL-22BP during DSS colitis and showed that IL-22BP is crucial in the control of IL-22 proliferative effects on epithelial cells. This control was particularly important to limit the tumorigenesis in a chronic colitis-associated colon cancer model. Importantly, the authors demonstrated that the increased tumorigenesis in IL-22BP $\mathrm{BP}^{-1-}$ mice was directly linked to the lack of IL-22 control, leading to prolonged deleterious actions of the cytokine. Thus, the authors showed, for the first time, the need for a tight regulation exerted by IL-22BP on IL-22 in vivo. However, IL-22BP ${ }^{-1-}$ mice did not show any phenotype in the steady state thus questioning the significance of constitutive IL-22BP expression in lymphoid tissues and intestine. Moreover, the authors also suggested in their study that DCs represent the major source of IL-22BP in mouse colon, a finding we confirmed here and further detailed by showing that only a subset of $\mathrm{CDCs}$ was expressing IL-22BP.
These data indicate that a tight, timely, and local regulation of IL-22 actions, likely by IL-22BP-expressing DCs, is critical in inflammatory conditions. Nonetheless, IL-22 also has a crucial role in the protection of the host from gut pathogens. ${ }^{5,6}$ During infection, strong upregulation of IL-22 is observed. IL-23 seems to be the main inducer of IL-22 production, principally by ILCs, which constitutively express IL-23R. ${ }^{47}$ Interestingly, mouse $\mathrm{CD}_{103}{ }^{+} \mathrm{CD}_{11 \mathrm{~b}}{ }^{+}$SI-LP DCs were recently shown to mediate rapid IL-22 production by ILC upon TLR5 triggering, through production of IL-23. ${ }^{47} \mathrm{CD}_{103}{ }^{+} \mathrm{CD} 1 \mathrm{~b}^{+}$DCs also promote the differentiation of $\mathrm{T}$ heper type 17 cells, which are known to produce IL-22, ${ }^{14}$ after TLR5 stimulation. ${ }^{48}$ Interestingly, TLR5 expression is a unique feature of these cells and also appears to be RA dependent. ${ }^{49}$ Combined with our data, this strongly suggests that the same subset is also the major source of IL-22BP in the intestine in the steady state, therefore reinforcing the central role of this DC subset in the control of IL-22. Finally, this subset of mouse $\mathrm{CD} 103^{+}$DCs was recently proposed to be the source of IL-23 in the thymus after injury. Again, IL-23 induced IL-22 production by ILCs. This IL-22 production was crucial for thymic recovery after injury but not for thymic development. ${ }^{50}$ IL-22BP expression by these cells was not explored but the low levels of IL-22BP expression in the thymus compared with SLO and gut suggest that the supposed IL-22BP production by $\mathrm{CD} 103^{+}$thymic DCs would probably be less important.

In conclusion, our study identified a subset of immature cDCs as a constitutive source of IL-22BP in the steady state in SLO and gut. Together with the recent report from Huber et al., these data strongly suggest that DC-produced IL-22BP has an important role in controlling IL-22 effect on epithelial cells. Moreover, our study suggests that the regulation of IL-22 by IL-22BP in the gut could be enhanced by RA. An unsolved crucial point concerns the constitutive secretion of IL-22BP by DCs that has never been demonstrated so far. The lack of an accurate and sensitive enough assay impeded our measurement of IL-22BP levels in culture supernatants. A better understanding of IL-22BP protein production and regulation by DCs and its importance in IL-22 regulation in the gut is necessary to establish the relationship between the observed beneficial and deleterious effects of the cytokine. It will also be important to confirm these data in human. This could be of importance in understanding the role played by IL- 22 in inflammatory bowel diseases, which still remains ambiguous today. ${ }^{8,9,51}$

\section{METHODS}

Animals. Sprague Dawley (SPD) rats were obtained from the Centre d'Elevage Janvier (Le Genest-St Isle, France) and were used when 6-10-weeks old. All animal experiments were performed under specific pathogen-free conditions in accordance with the European Union Guidelines. All animal studies were conducted according to the guidelines of the French Agriculture Ministry. The studies were approved by the Veterinary Departmental Services committee (No. E.44011).

Reagents. Cells were cultured in complete RPMI 1640 (Invitrogen, Carlsbad, CA). Recombinant human GM-CSF, IL-4, and IL-10 were 
from CellGenix (Fribourg-en-Brisgau, Germany), recombinant human TGF- $\beta$ and IL-13 were from Peprotech (Neuilly-sur-Seine, France), recombinant IFN- $\gamma$ was from R\&D Systems Europe (Lille, France). $\mathrm{PGE}_{2}$, RA, AM580, retinal, DEAB, and LPS were from Sigma Aldrich (St Louis, MI). CpG ODN2006 was from Eurofins MWG Operon (Ebersberg, Germany). Selective RA nuclear receptor inhibitors BMS 195314 (RAR $\alpha$ inhibitor), UVI 3003 (retinoic X receptor inhibitor), and CD 2665 (RAR $\beta / \gamma$ inhibitor) were from Tocris bioscience (Lille, France).

Cell sorting. Rat splenic cells. Rat conventional and plasmacytoid DCs subsets were isolated as previously described. ${ }^{2,52,53}$ For lymphocytes and monocytes, cells were first separated by Ficoll-Paque Plus (GE HealthCare Life Sciences, Uppsala, Sweden) gradient centrifugation. For lymphocytes, cells were stained with T-cell receptor- $\alpha \beta$ (TCR $\alpha \beta$ )-biotin (clone R7.3), CD45R-phycoerythrin (PE; clone HIS24), CD45RA-FITC (fluorescein isothiocyanate; clone OX33), NKR-P1-Alexa647 (clone 3-2-3) mAbs, and then with streptavidin-PE-Cy7. Live TCR $\alpha \beta^{+}$(T cells), TCR $\alpha \beta^{-}$ CD45R ${ }^{+} \mathrm{CD}_{45 \mathrm{RA}}{ }^{+} \mathrm{NKR}^{-\mathrm{P}} 1^{-}$(B cells), and TCR $\alpha \beta^{-} \mathrm{CD}^{-} 5 \mathrm{R}^{-}$ $\mathrm{CD}^{2} 5 \mathrm{RA}^{-}{ }^{-} \mathrm{NKRP}^{+}{ }^{+}$(NK cells) were Facs-sorted. For monocytes, cells were stained with MHC II-PE (clone OX6), CD172 $\alpha$-biotin (clone OX41), CD103-Alexa647 (clone OX62), and CD11b/cAlexa488 (clone OX42) mAbs, and then with streptavidin PE-Cy7. Live $\mathrm{CMH}-\mathrm{II}^{+} \mathrm{CD}^{+} 3^{-} \mathrm{CD} 172 \alpha^{+} \mathrm{CD} 11 \mathrm{~b} / \mathrm{c}^{+}$(monocytes) were Facs-sorted.

Rat ilDCs. Lymph was collected from male animals using previously described methods. ${ }^{28,29}$ Briefly, mesenteric lymphadenectomy was performed on 5-6-week-old animals. At least 5 weeks later, a cannula (3Fr, Harvard Apparatus, Kent, UK) was inserted into the thoracic duct at laparotomy. Lymph was collected for up to $48 \mathrm{~h}$ on ice. Red blood cells were lysed from lymph, and the single-cell suspensions were enriched for DCs using magnetic beads specific for CD103 (Miltenyi Biotec, Paris, France) according to the manufacturer's protocol. Enriched cells were stained with antibodies for MHCII (OX-6, BD Biosciences, Le Pont-de-Claix, France), CD103 (OX-62, in house), CD11b (OX-42, BD Biosciences), and CD172a (OX-41, in house), and the three L-DC subsets were flow sorted using BD FACS Aria Cell sorter. RNA was isolated from sorted cells using Qiagen RNeasy Mini Kit according to the manufacturer's instructions. Complementary DNA (cDNA) was reverse-transcribed from RNA using Superscript First-Strand Synthesis System for reverse transcriptase-PCR (Invitrogen).

Rat MLN cDCs. MLN were harvested from adult SPD rats, dilacerated using $26 \mathrm{G}$ needles and digested for $25 \mathrm{~min}$ with Collagenase $\mathrm{D}$, in the presence of DNAse I. Cells were collected and low density cells were prepared using a $14,5 \%$ Nycodenz gradient. Cells were then stained with anti-TCR $\alpha \beta$ (clone R7/3), anti-CD45R (clone HIS24), antiCD103 (clone OX62) and anti-CD4 (clone W3/25) and sorted on a FACS Aria into TCR $\alpha \beta-$ CD $45 \mathrm{R}-\mathrm{CD} 103+\mathrm{CD} 4+$ /int $/$ - cDCs. Purity was routinely $>95 \%$.

Mouse LP cells. SIs were flushed with HBSS (Hank's Balanced Salt Solution) 2\% fetal calf serum and the Peyer's patches excised. The intestines were opened longitudinally and cut into $0.5-\mathrm{cm}$ segments, which were incubated twice in HBSS with $2 \mathrm{~mm}$ EDTA at $37^{\circ} \mathrm{C}$ while shaking for $20 \mathrm{~min}$. Supernatants were discarded and the tissue digested with $1 \mathrm{mg} \mathrm{ml}^{-1}$ of collagenase VIII (Sigma-Aldrich) at $37^{\circ} \mathrm{C}$ with shaking for $15 \mathrm{~min}$. Cells were passed through a $40-\mu \mathrm{m}$ cell strainer and stained for flow cytometry. DCs (Live CD $45^{+}$

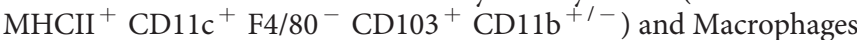
(Live $\mathrm{CD}^{+} 5^{+} \mathrm{MHCII}^{+} \mathrm{F} 4 / 80^{+} \mathrm{CD} 11 \mathrm{~b}^{+}$) were sorted using a FACSAria I (purity was routinely $>95 \%$ ).
Mouse MLN CD103 + cDCs. MLN were digested using liberase (0.4 Wunsch Units $\mathrm{ml}^{-1}$; Roche, Meylan, France) and DNAse $\left(50 \mu \mathrm{g} \mathrm{ml}^{-1}\right.$; Roche) for $45 \mathrm{~min}$ at $+37^{\circ} \mathrm{C}$. Single-cell suspensions were stained for flow cytometry with MHCII IA/IE (clone M5/ 114.15.2) antibody from ebioscience (San Diego, CA) and CD11b (clone M1/70), B220 (clone RA3-6B2), CD103 (clone 2E7), CD11c (clone N418) antibodies from Biolegend (London, UK).

Human cell preparation. Human blood was obtained from healthy donors upon informed consent in accordance with our Institutional Review Board. Peripheral blood mononuclear cells were separated by density gradient centrifugation over Ficoll (PAA, Pasching, Austria). $\mathrm{CD}_{14}{ }^{+}$monocytes were isolated by positive selection (Miltenyi Biotec) and differentiated into DCs with $1,000 \mathrm{U} \mathrm{ml}^{-1} \mathrm{GM}-\mathrm{CSF}$ and $200 \mathrm{U} \mathrm{ml}^{-1} \mathrm{IL}-4$ for 6 days. When indicated, cells were treated with AM580 or RA (100 nM), IL-10 (50 $\left.\mathrm{ng} \mathrm{ml}^{-1}\right)$, IL-13 $\left(10 \mathrm{ng} \mathrm{ml}^{-1}\right)$, TGF- $\beta$ (10 $\left.\mathrm{ng} \mathrm{ml}^{-1}\right), \mathrm{PGE}_{2}\left(10 \mathrm{ng} \mathrm{ml}^{-1}\right)$, retinal (100 nM), DEAB $(150 \mu \mathrm{M})$, LPS $\left(1 \mu \mathrm{g} \mathrm{ml}^{-1}\right), \quad$ IFN- $\gamma\left(50 \mathrm{ng} \mathrm{ml}^{-1}\right)$, BMS 195314 $\left(5 \mu \mathrm{mol} \mathrm{ml}{ }^{-1}\right)$, UVI $3003\left(5 \mu \mathrm{moll}^{-1}\right)$, or CD $2665\left(5 \mu \mathrm{moll}{ }^{-1}\right)$.

Flow cytometry analysis. Human MDDCs were stained with HLADR-APC (clone L243 (G46-6)), CD209-PE (clone DCN46), CD1d-PE (clone CD1d42) and CD103-FITC (cloneBer-ACT8), all from $\mathrm{BD}$ Biosciences, or isotype-matched control antibodies. Cells were analyzed on a BD FACSCanto II flow cytometer (BD Biosciences). Data were analyzed using FlowJo software (Treestar, Ashland, OR).

Real-time quantitative reverse transcriptase-PCR. Total RNA was isolated using Trizol reagent (Invitrogen) or Qiagen RNeasy Mini Kit according to the manufacturers' instructions. Reverse transcription was performed using Murine Moloney Leukemia Virus Reverse Transcriptase (Invitrogen) or Superscript First-Strand Synthesis System for reverse transcriptase-PCR (Invitrogen), following the manufacturer's instructions. For rat IL-22BP, IL-22RA1, ALDH1A2, mouse IL-22BP, and human ALDH1A2 gene expression, Power Sybr Green $2 \times$ reagent was used (Applied Biosystems, Foster City, CA). Real-time PCR was performed using the Viia 7 Real Time PCR system (Applied Biosystems). Primers (Eurofins MWG Operon, Ebersberg, Germany) are summarized in Supplementary Table S1. For human IL-22BP gene expression, TaqMan Fast Advanced Master Mix $2 \times$ reagent was used (Applied Biosystems). Primers and probes were from Applied Biosystems. Real-time PCR was performed using the StepOne Plus system (Applied Biosystems). For both human and rat, relative expression was normalized from HPRT (hypoxanthineguanine phosphoribosyltransferase) and calculated using the $2^{-\Delta \Delta \mathrm{Ct}}$ method. For mouse, relative expression was normalized from GAPDH (glyceraldehyde 3-phosphate dehydrogenase). Results were expressed in arbitrary units.

Whole IL-22BP cDNA amplification. In order to identify IL-22BP isoforms, whole cDNA amplification was performed in both human and rat samples. Primers used for rat and human IL-22BP are summarized in Supplementary Table S2. Amplification was performed using HERCULASE II Fusion Enzyme (Agilent, Santa Clara, CA).

Indirect immunofluorescence. Cells were let to adhere on polyL-Lysine pre-coated slides for $30 \mathrm{~min}$ (Sigma Aldrich). Fixation was performed for $5 \mathrm{~min}$ in $-20{ }^{\circ} \mathrm{C}$ pre-cold acetone. After rehydration, a 30 min step saturation was made with phosphate-buffered saline/ bovine serum albumin 1\%/serum $10 \%$. Primary antibody (antiIL-22BP from R\&D Systems for human MDDCs or anti-rat IL-22BP from Santa Cruz Biotechnology, Santa Cruz, CA for rat cDCs) was incubated at room temperature for $2 \mathrm{~h}$. Secondary antibodies were then incubated for $1 \mathrm{~h}$ at room temperature.

Statistical analysis. Data are represented as means \pm s.e.m. Statistical analysis was performed with GraphPad Prism Software (GraphPad Software, San Diego, CA). Means comparisons were performed using 
the Mann-Whitney $U$ test or the Kruskal-Wallis test with Dunn's post test. $P$-values $<0.05$ were considered statistically significant.

SUPPLEMENTARY MATERIAL is linked to the online version of the paper at http://www.nature.com/mi

\section{ACKNOWLEDGEMENTS}

We thank Professor JC Renauld (Université Catholique de Louvain, Belgium) for critically reading the manuscript.

\section{DISCLOSURE}

The authors declare no conflict of interest.

(c) 2014 Society for Mucosal Immunology

\section{REFERENCES}

1. Sonnenberg, G. F., Fouser, L. A. \& Artis, D. Border patrol: regulation of immunity, inflammation and tissue homeostasis at barrier surfaces by IL22. Nat. Immunol. 12, 383-390 (2011).

2. Wolk, K. et al. IL-22 increases the innate immunity of tissues. Immunity $\mathbf{2 1}$, 241-254 (2004).

3. Radaeva, S., Sun, R., Pan, H.-N., Hong, F. \& Gao, B. Interleukin 22 (IL-22) plays a protective role in Tcell-mediated murine hepatitis: IL-22 is a survival factor for hepatocytes via STAT3 activation. Hepatology 39, 1332-1342 (2004).

4. Aggarwal, S., Xie, M. H., Maruoka, M., Foster, J. \& Gurney, A. L. Acinar cells of the pancreas are a target of interleukin-22. J. Interferon Cytokine Res. 21 1047-1053 (2001).

5. Zheng, Y. et al. Interleukin-22 mediates early host defense against attaching and effacing bacterial pathogens. Nat. Med. 14, 282-289 (2008)

6. Aujla, S. J. et al. IL-22 mediates mucosal host defense against Gram-negative bacterial pneumonia. Nat. Med. 14, 275-281 (2008).

7. Simonian, P. L. et al. $\gamma \delta$ Tcells protect against lung fibrosis via IL-22. J. Exp. Med. 207, 2239-2253 (2010).

8. Sugimoto, K. et al. IL-22 ameliorates intestinal inflammation in a mouse model of ulcerative colitis. J. Clin. Invest. 118, 534-544 (2008).

9. Zenewicz, L. A. et al. Innate and adaptive interleukin-22 protects mice from inflammatory bowel disease. Immunity 29, 947-957 (2008).

10. Zheng, Y. et al. Interleukin-22, a T(H)17 cytokine, mediates IL-23-induced dermal inflammation and acanthosis. Nature 445, 648-651 (2007).

11. Geboes, L. et al. Proinflammatory role of the Th17 cytokine interleukin-22 in collagen-induced arthritis in C57BL/6 mice. Arthritis Rheum. 60, 390-395 (2009).

12. Muñoz, M. et al. Interleukin (IL)-23 mediates Toxoplasma gondii-induced immunopathology in the gut via matrixmetalloproteinase-2 and IL-22 but independent of IL-17. J. Exp. Med. 206, 3047-3059 (2009).

13. Sonnenberg, G. F. et al. Pathological versus protective functions of IL-22 in airway inflammation are regulated by IL-17A. J. Exp. Med. 207, 1293-1305 (2010).

14. Liang, S. C. et al. Interleukin (IL)-22 and IL-17 are coexpressed by Th17 cells and cooperatively enhance expression of antimicrobial peptides. J. Exp. Med. 203, 2271-2279 (2006).

15. Dumoutier, L., Lejeune, D., Colau, D. \& Renauld, J. C. Cloning and characterization of IL-22 binding protein, a natural antagonist of IL-10related T cell-derived inducible factor/IL-22. J. Immunol. 166, 7090-7095 (2001).

16. Kotenko, S. V. et al. Identification, cloning, and characterization of a novel soluble receptor that binds IL-22 and neutralizes its activity. J. Immunol. 166, 7096-7103 (2001).

17. Xu, W. et al. A soluble class II cytokine receptor, IL-22RA2, is a naturally occurring IL-22 antagonist. Proc. Natl. Acad. Sci. USA 98, 9511-9516 (2001).

18. Logsdon, N. J., Jones, B. C., Josephson, K., Cook, J. \& Walter, M. R. Comparison of interleukin-22 and interleukin-10 soluble receptor complexes. J. Interferon Cytokine Res. 22, 1099-1112 (2002).

19. Wolk, K. et al. IL-22 induces lipopolysaccharide-binding protein in hepatocytes: a potential systemic role of IL-22 in Crohn's disease. J. Immunol. 178, 5973-5981 (2007).
20. De Moura, P. R. et al. Crystal structure of a soluble decoy receptor IL-22BP bound to interleukin-22. FEBS Lett. 583, 1072-1077 (2009).

21. Banchereau, J. \& Steinman, R. M. Dendritic cells and the control of immunity. Nature 392, 245-252 (1998).

22. Shortman, K. \& Naik, S. H. Steady-state and inflammatory dendritic-cell development. Nat. Rev. Immunol. 7, 19-30 (2007).

23. Villadangos, J. A. \& Schnorrer, P Intrinsic and cooperative antigenpresenting functions of dendritic-cell subsets in vivo. Nat. Rev. Immunol. 7, 543-555 (2007).

24. Weiss, B. et al. Cloning of murine IL-22 receptor alpha 2 and comparison with its human counterpart. Genes Immun. 5, 330-336 (2004).

25. Piriyapongsa, J., Polavarapu, N., Borodovsky, M. \& McDonald, J. Exonization of the LTR transposable elements in human genome. BMC Genomics 8, 291 (2007).

26. Trinité, B., Voisine, C., Yagita, H. \& Josien, R. A subset of cytolytic dendritic cells in rat. J. Immunol. 165, 4202-4208 (2000).

27. Turnbull, E. \& MacPherson, G. Immunobiology of dendritic cells in the rat. Immunol. Rev. 184, 58-68 (2001).

28. Milling, S. \& MacPherson, G. Isolation of rat intestinal lymph DC. Methods Mol. Biol. 595, 281-297 (2010).

29. Milling, S. W. F., Jenkins, C. \& MacPherson, G. Collection of lymph-borne dendritic cells in the rat. Nat. Protoc. 1, 2263-2270 (2006).

30. Milling, S., Yrlid, U., Cerovic, V. \& MacPherson, G. Subsets of migrating intestinal dendritic cells. Immunol. Rev. 234, 259-267 (2010).

31. lliev, I. D. et al. Human intestinal epithelial cells promote the differentiation of tolerogenic dendritic cells. Gut 58, 1481-1489 (2009).

32. Szatmari, I. et al. PPARgamma controls CD1d expression by turning on retinoic acid synthesis in developing human dendritic cells. J. Exp. Med. 203, 2351-2362 (2006).

33. Iwata, M. \& Yokota, A. Retinoic acid production by intestinal dendritic cells. Vitam. Horm. 86, 127-152 (2011)

34. Stock, A., Booth, S. \& Cerundolo, V. Prostaglandin E2 suppresses the differentiation of retinoic acid-producing dendritic cells in mice and humans. J. Exp. Med. 208, 761-773 (2011).

35. Huber, S. et al. IL-22BP is regulated by the inflammasome and modulates tumorigenesis in the intestine. Nature 491, 259-263 (2012).

36. Weber, G. F. et al. Inhibition of interleukin-22 attenuates bacterial load and organ failure during acute polymicrobial sepsis. Infect. Immun. 75, 1690-1697 (2007)

37. Wilson, M. S. et al. Redundant and pathogenic roles for IL-22 in mycobacterial, protozoan, and helminth infections. J. Immunol. 184, 4378-4390 (2010)

38. Jiang, A. et al. Disruption of E-cadherin-mediated adhesion induces a functionally distinct pathway of dendritic cell maturation. Immunity 27 , 610-624 (2007).

39. Merad, M., Sathe, P., Helft, J., Miller, J. \& Mortha, A. The dendritic cell lineage: ontogeny and function of dendritic cells and their subsets in the steady state and the inflamed setting. Annu. Rev. Immunol. 31, 563-604 (2013).

40. Gelebart, P., Zak, Z., Dien-Bard, J., Anand, M. \& Lai, R. Interleukin 22 signaling promotes cell growth in mantle cell lymphoma. Transl. Oncol. 4, 9-19 (2011).

41. Savan, R. et al. A novel role for IL-22R1 as a driver of inflammation. Blood 117, 575-584 (2011)

42. Jaensson, E. et al. Small intestinal CD103 + dendritic cells display unique functional properties that are conserved between mice and humans. J. Exp. Med. 205, 2139-2149 (2008).

43. Scott, C. L., Aumeunier, A. M. \& Mowat, A. M. Intestinal CD103 + dendritic cells: master regulators of tolerance? Trends Immunol. 32, 412-419 (2011).

44. Agace, W. W. \& Persson, E. K. How vitamin A metabolizing dendritic cells are generated in the gut mucosa. Trends Immunol. 33, 42-48 (2011).

45. Sawa, S. et al. RORrt(+) innate lymphoid cells regulate intestinal homeostasis by integrating negative signals from the symbiotic microbiota. Nat. Immunol. 12, 320-326 (2011).

46. Eberl, G. Development and evolution of ROR $\gamma \mathrm{t}(+)$ cells in a microbe's world. Immunol. Rev. 245, 177-188 (2012). 
47. Kinnebrew, M. A. et al. Interleukin 23 production by intestinal CD103(+) $\mathrm{CD} 11 \mathrm{~b}(+)$ dendritic cells in response to bacterial flagellin enhances mucosal innate immune defense. Immunity 36, 276-287 (2012).

48. Uematsu, S. et al. Regulation of humoral and cellular gut immunity by lamina propria dendritic cells expressing Toll-like receptor 5. Nat. Immunol. 9, 769-776 (2008).

49. Cho, H.-Y. et al. All-trans retinoic acid induces TLR- 5 expression and cell differentiation and promotes flagellin-mediated cell functions in human THP-1 cells. Immunol. Lett. 136, 97-107 (2011).

50. Dudakov, J. A. et al. Interleukin-22 drives endogenous thymic regeneration in mice. Science 336, 91-95 (2012).
51. Brand, S. et al. IL-22 is increased in active Crohn's disease and promotes proinflammatory gene expression and intestinal epithelial cell migration. Am. J. Physiol. Gastrointest. Liver Physiol. 290, G827-G838 (2006).

52. Anjubault, T. et al. Constitutive expression of TNF-related activationinduced cytokine (TRANCE)/receptor activating NF-kB ligand (RANK)-L by rat plasmacytoid dendritic cells. PLoS ONE 7, e33713 (2012).

53. Hubert, F.-X., Voisine, C., Louvet, C., Heslan, M. \& Josien, R. Rat plasmacytoid dendritic cells are an abundant subset of MHC class II+ CD4 + CD11b-OX62- and type I IFN-producing cells that exhibit selective expression of Toll-like receptors 7 and 9 and strong responsiveness to CpG. J. Immunol. 172, 7485-7494 (2004). 